\title{
Using Side Scan Sonar to Relative Navigation
}

\author{
Miguel Pinto, Bruno Ferreira, Aníbal Matos, Nuno Cruz \\ FEUP-DEEC, Rua Dr. Roberto Frias, 4200-465 Porto, Portugal \\ ee04134@fe.up.pt, ee04018@fe.up.pt,anibal@fe.up.pt,nacruz@fe.up.pt
}

\begin{abstract}
This paper describes the interaction between the kinematic model of the AUV MARES and the measurement or observation of the environment through images obtained with a sonar. Three types of sonar are discussed in this paper: forwardlook, side scan and multibeam - but the sonar used to develop this work was the side scan sonar. The type of observations and characteristics of the environment provided by the sonar are described here. The method which connects the sensory part of the vehicle with the observations from the sonar, was the Kalman filter (EKF). In this paper, we present two simulations of filters for two different characteristics. Both filters estimate the characteristics of the natural landmarks, creating an environment map, but both of them consider different states of the vehicle. Results of the simulation are obtained. The features that are considered are an underwater pipe on the floor and a vertical wall. A control loop for the vehicle that provides the capacity to move along the feature/landmark from a reference distance is also discussed.
\end{abstract}

\section{INTRODUCTION}

Some vehicles, such as ROVs (remotely operated vehicles) are not able to operate without a power cable. Other vehicles such as AUVs (Autonomous Underwater Vehicles) are not able to navigate without the help of methods like absolute location, such as GPS or acoustic beacons. In fact, these vehicles have a certain degree of autonomy, but cannot be considered as truly autonomous [1],[2],[3],[4] and [5].

The true definition of autonomy is the capacity that the vehicle has to move and locate in the world, independently, with the maximum certainty of localization and without environment preparation [6]. One of the fundamental characteristics of autonomy is also the capacity of the vehicle moving without bounds, often introduced by methods such as acoustic beacons, which have a maximum range.

It is this definition of autonomy that is the opportunity of this work. The fundamental aim is to equip the AUV MARES [3] with real autonomy by using side scan sonar, allowing it to navigate relative to landmarks in the marine environment, such as pipes and walls.

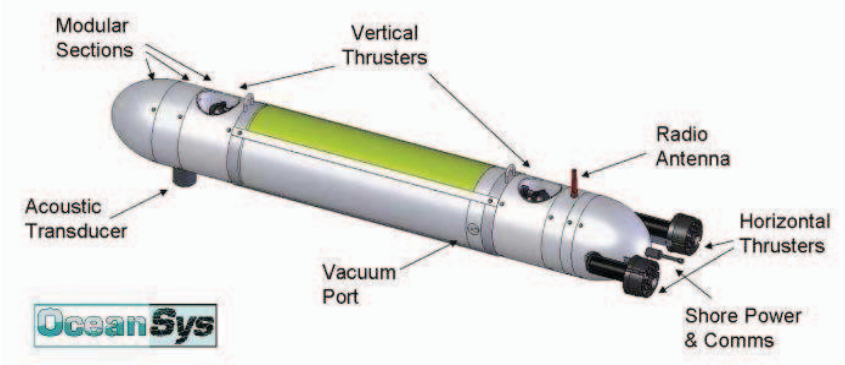

Figure 1 AUV MARES developed by the OceanSys Group FEUP-DEEC.
The importance of providing a higher degree of autonomy to vehicles appears because the use of AUVs has had a rapid growth both on civil and military applications in recent years.

In section II sensors and navigation methods are described. The third section describes sonar data, and a computational method to extract the features. In section IV the marine bottom algorithm is applied to a real acoustic image, while in the fifth section two navigation filters for two different features are presented. In section VI the operation limits of side scan sonar are studied. In section VII AUV guidance and control are described. Finally in section VIII simulation results are presented.

\section{UNDERWATER VEHICLES AUTONOMY}

The autonomy in ocean robotic is divided into the following three levels, [5]:

- Self-energy: provides a vehicle own sources of energy. - Self-navigation: represents the capacity of the vehicle to navigate accurately with a low error of estimation. This type of autonomy is the fundamental unit of study this paper. - Autonomy of decision: this is the ability to decide and act in various scenarios.

\section{A. Evolution of Autonomy in Underwater Robotics}

Different sensors and techniques for localization of vehicles are described in [7]. These sensors and techniques are divided into absolute and relative localization.

Dead-reckoning sensors, such as inertial navigation (INS), attitude sensors, digital compass, and Doppler-effect sensor (DVL), are relative sensors of localization in space. The position of the vehicle in the world is given by the sum of successive estimated position differences, leading to a position error that grows without bounds.

In absolute localization, at each instant of time, the position of the vehicle in the world is directly estimated. Some methods of absolute localization are based on active beacons, such as acoustic beacons LBL (long baseline), ULBL (Ultra long baseline) and SBL (Short Baseline) and Global Positioning System (GPS or DGPS).

The methods based on acoustic beacons and the GPS involve the need for additional logistics or high errors of localization [7]. Or the other hand, terrain-based navigation methods combine relative and absolute navigation methods, and resort to landmarks existing on the sea environment.

B. SLAM-Simultaneous Localization and Mapping

Terrain-based navigation [8] based on natural landmarks has essentially two phases:

-Construction Map, knowing the location of the vehicle in the world by other methods of localization.

-Localization of the vehicle based on the world map constructed a priori. 
The aim of this work, using side scan sonar and using the sensors of attitude and dead-reckoning installed on the vehicle, is to join the two stages of the terrain navigation on a single stage, allowing the vehicle to construct the map and to locate itself simultaneously. This is a problem usually known as SLAM (Simultaneous Localization and Mapping) or CLM (Concurrent Localization and Mapping).

Both SLAM and CLM have the same objective and contribute to the true autonomy of a robotic platform. The problem of SLAM is treated in [6], applied on AGVs (Autonomous ground vehicles), while CML is treated in [8] on AUVs.

Using a SLAM/CML based methods the AUV will have autonomy and can be launched on missions to search and collect data with minimal preparation, without bounds of navigation and with a great certainty of position estimation.

To make the interconnection of relative navigation methods, dead-reckoning, with observations obtained by sonar, a method as the Kalman filter is needed.

The Kalman filter, more precisely EKF (Extended Kalman Filter), described in [9], consists of a Kalman filter in which the state vector consists not only on information about the vehicle (its position), but also on information detected and identified from natural landmarks. Thus, the estimation will be not only the vehicle but also the environment, which in fact results on a SLAM problem. The approach described here follows the ideas from [6], [8], [10], [11] and [12] where Kalman filters are also employed.

\section{SONAR DATA}

Sonar can be used to obtain acoustic images of the sea bed, allowing the extraction an identification of natural landmarks.

The side scan sonar comparative with forward-look sonar gives more quality images from sea bed. Thus it provides better and easily extraction of features [8].

An image obtained by side scan sonar is composed by the echo strengths along lines normal to the motion of the sonar. The image is formed line by line as the sonar moves, ideally in a liner motion.

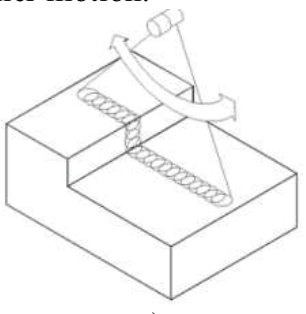

a)

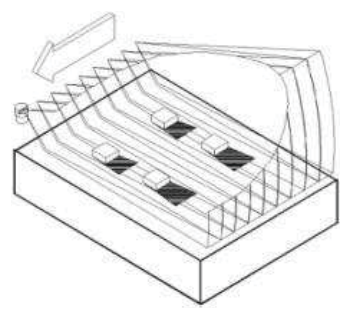

b)
Figure 2 a) Forward-look scan, [13]. b) Side scan, [13].

In this work the chosen sonar was side scan sonar, more precisely, the Imaginex Sportscan, that is shown in figure 2. It follows some ideas presented in [8] and [9], where a side scan sonar as also employed.

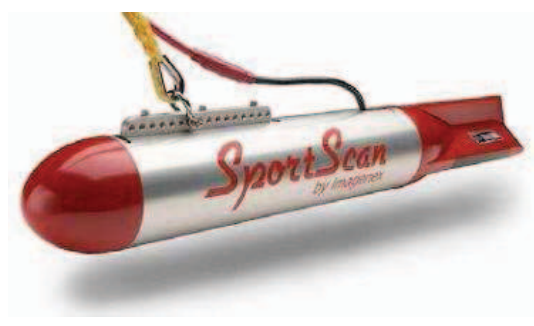

Figure 3 Side scan Imagenex Sportscan, [13].

Other sonar type is the multibeam sonar. It corresponds to a sonar type in which the quality of acoustic image obtained is better than side scan or forward-look. The information collected is larger, which allows terrain-based navigation of higher quality. The multibeam sonar, gives a threedimensional pictures of the environment. It is composed by an array of sonars (multibeam), arranged in the same direction and angle of incidence fixed, known and that differs between them. Nonetheless, these sonars are much heavier and consume more power, making them less attractive for small size AUV applications, as the one addressed here.

\section{A. Obtained Data by Analisys of Acoustic Image}

For a feature located at the sea floor, the analysis of an acoustic image provides important data required for the navigation procedure described here. That data are:

-The size of the no-echo zone, $(\mathrm{H})$ allows us to know the height above the floor at which the AUV is.

-A sum of no-echo zone with the area of the bottom echo and the target, $(\mathrm{H}+\mathrm{r})$, allows to know the distance between the submarine and the target.

-A shadow zone of the target (S), where there is no-echo, lets us know the height of the target. This height is given by the expression:

$$
h=\frac{H . S}{r+S}
$$

All these characteristics can be obtained by image processing algorithms.

The algorithms of segmentation and extraction of features should be flexible and then be able to handle images whose quality is lower than the figures 4 , a) and b).

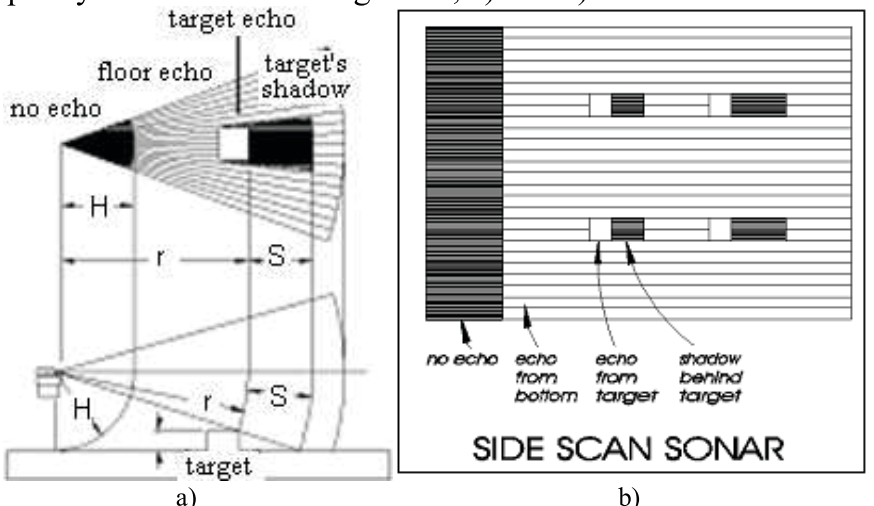

a)

b)

Figure 4 a) Beam Observation, Target values (H, r, h and s), [13]. b) Side scan image, [13]. 


\section{B. Data segmentation and extraction}

The image obtained by the SportScan sonar is a grey scale image (monochromatic image). The image size is equals to $500 \times$ N. Each slice acquired from Imagenex SportScan has 500 points of data. $\mathrm{N}$ corresponds to a number of slices that constitute de image.

To obtain sea floor detection, the sequences of image operations applied are:

-Detect the minimum value of the picture and thus non-echo zone (black pixels).

-A unimodal threshold is applied with the goal to transform the grey scale image on binary image. Using the minimum value as threshold value the sea floor continues black and the echo from bottom and targets become white, including the surface echo.

-Dilate followed by erode are applied with a structured element with square shape with $3 \times 3$ dimension to eliminate small holes (black pixels) into the bottom echo zone (white pixels).

-Erode followed by dilate are applied with a structured element also with square shape and 10x10 dimension to clear thin lines from surface echo into the non-echo zone (black pixels).

-Horizontal edge detection can be accomplished by applying the square matrix with dimensions $3 \times 3$, Prewitt filter:

$$
\text { Prewitt }=\left[\begin{array}{ccc}
-1 & -1 & -1 \\
0 & 0 & 0 \\
1 & 1 & 1
\end{array}\right]
$$

The convolution of this filter with the image is equal to zero when applied in homogeneous zones and it is different from zero when applied on edge pixels. The convolution result is an image with edges detected and represented by a straight line, figure $6 \mathrm{~b}$ ) (right).

-Finally the Hough transform is employed to obtain line equations.

To extract feature equations like a pipe or a wall, the procedure is the same with the difference that the threshold value is now equal to the maximum value of the picture and thus equals to echo feature zone (more white pixels).

All these image methods and computational processes can be seen on [14], [15] and [16].

\section{Sliding Window}

Using a sliding window, possible disturbances in the image can be reduced. Each slice acquired by sonar has random noise that results from the acquisition process. This requires the use of a sliding window to decrease the error measurements due to random noise in sonar data.

Thus, in each sampling instant, the observation will be the new slice acquired by side scan added to the others slices, according to the sliding window size.

The sliding window length cannot be very large otherwise the observations would correspond to features observed at a large distance from the vehicle actual position.

So, a trade-off on sliding window length has to be made and it must be larger as possible.

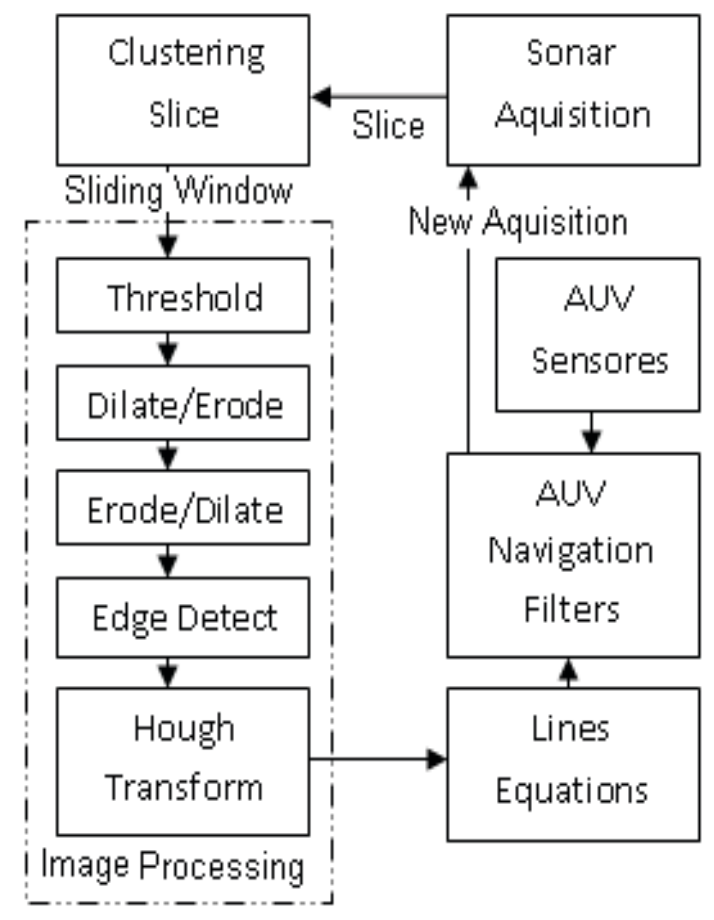

Figure 5 Algorithm to obtain the sea floor equation, starboard and port, and the interaction of lines information with a possible AUV navigation.

To determine the sliding window size, the time of acquisition has to be considered. The request frame for sonar data is $B_{\text {req }}$ bytes long. The sonar response length is $B_{\text {res }}$. The acquisition time of data is equal to the time between acoustic impulse transmission and the respective echo arrivals. Considering the data exchange between the vehicle and sonar based on a RS-232 link, the total time acquisition of a slice is given by:

$$
T=B R \cdot B i t s\left(B_{r e q}+B_{r e s}\right)+2 \frac{\text { Range }}{v},
$$

where $B R$ is de baud rate, Bits the number bits per byte, Range the sonar reach in meters and $v$ is the velocity of propagation of the sound in water.

If the goal is to follow a path parallel to the feature, as an underwater pipe on the floor or a wall, the window size will be:

$$
w_{\text {size }}=\frac{\operatorname{Dmax}}{V \cdot T}
$$

where Dmax corresponds the maximum distance between the sonar and the feature on the orthogonal direction; D max will be equal to an image pixel size; and $V$ corresponds to vehicle speed on the direction orthogonal to the feature.

\section{Real Image Sea Floor Detection}

The extraction of features, which in that case, figure 6 a), only correspond to sea floor, was made using a sliding window with fixed size of 10 slices, Figure 6 b) (left). 


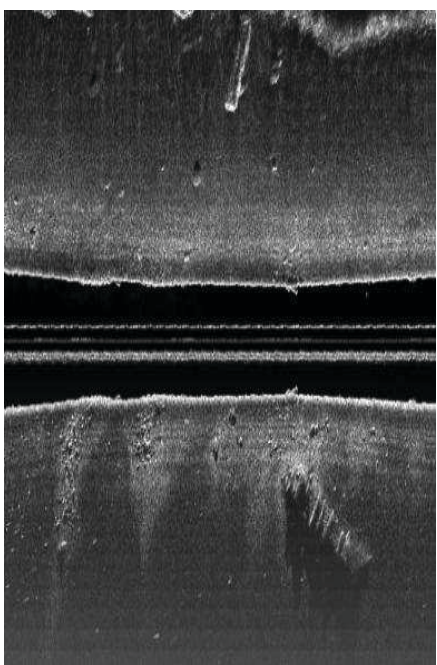

a)

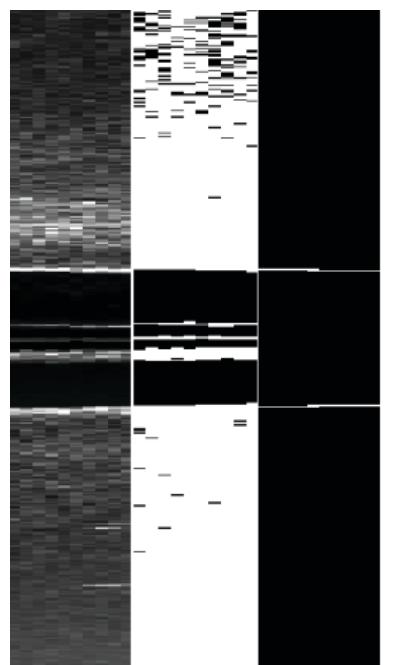

b)
Figure 6 a) Real acoustic image, shows the perception of the sea bed. It is possible see when the echo from the floor and surface appears. b) Results. Original sliding window (left). Intermediate result from segmentation (middle). Final result (right).

\section{NAVIGATION FILTERS}

\section{A. Kalman Filter: State (East, South, Depth)}

To estimate the position of the vehicle, two reference frames are considered: a static world fixed frame and a moving frame attached to the vehicle [17], as shown in figure 7.

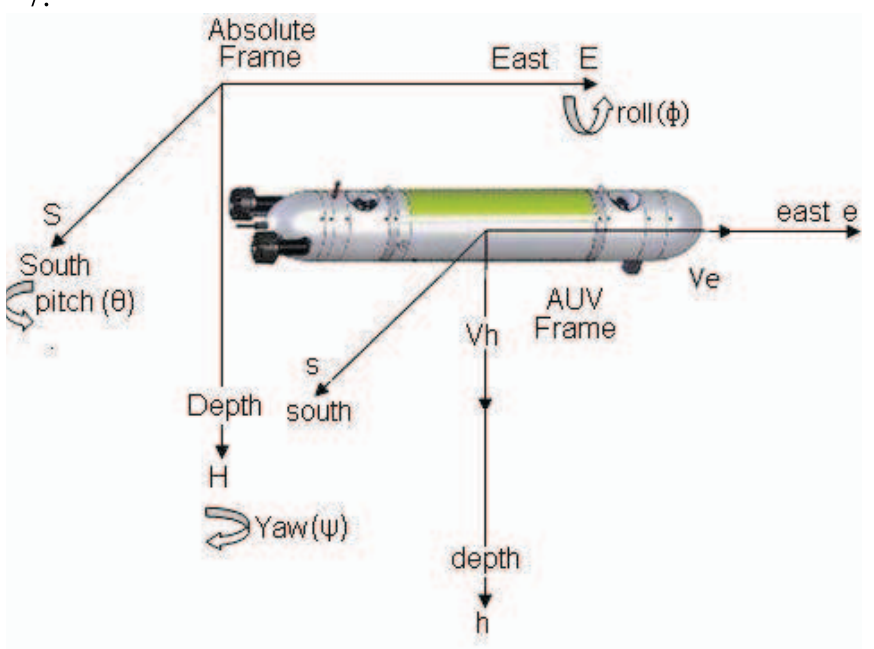

Figure 7 Absolute frame and AUV frame.

Considering a straight line feature characterized by a line of slope $(\mathrm{m})$ and origin intersection (b), on sea bed, the process model of the filter will be given by a non linear system in continuous time. The state vector is:

$$
x=\left[\begin{array}{lllll}
E_{v} & S_{v} & H_{v} & m & b
\end{array}\right]^{T}
$$

The linear speed of the vehicle only exists in two directions, east (e) and depth (h) axis. The kinematic model of the vehicle will be given by:

$$
\dot{x}(t)=\left[\begin{array}{c}
c \psi(t)\left(c \theta(\mathrm{t}) V_{e}(t)+\mathrm{s} \theta(\mathrm{t}) V_{h}(t)\right) \\
\mathrm{s} \psi(t)\left(\mathrm{c} \theta(\mathrm{t}) V_{e}(t)+\mathrm{s} \theta(\mathrm{t}) V_{h}(t)\right) \\
-\mathrm{s} \theta(\mathrm{t}) V_{e}(t)+c \theta(\mathrm{t}) V_{h}(t) \\
0 \\
0
\end{array}\right]
$$

Where $c(\cdot)$ is $\cos (\cdot)$, and $s(\cdot)$ is $\sin (\cdot)$. Along this paper that notation will be used.

The input vector $u(t)$ and the Gaussian white noise $w(t)$, with mean zero, associated with each input, are represented by:

$$
\begin{gathered}
u=\left[\begin{array}{cccc}
V_{e v} & V_{h v} & \theta_{v} & \psi_{v}
\end{array}\right]^{T}, \\
w=\left[\begin{array}{llll}
\epsilon_{V e} & \epsilon_{V h} & \epsilon_{\theta_{v}} & \epsilon_{\psi_{v}}
\end{array}\right]^{T} \\
{[w(k)]=0 \text { and } E\left[w(k) w^{T}(k)\right]=Q}
\end{gathered}
$$

\section{B. Sonar Observation: Feature Pipe.}

Each time instant, the vehicle, detect a point of the pipe, on its frame. Then each observation is the point:

$$
X i=\left[\begin{array}{lll}
e_{T} & s_{T} & h_{T}
\end{array}\right]^{\mathrm{T}}=\left[\begin{array}{lll}
0 & \sqrt{r^{2}-h_{T}{ }^{2}} & H c \beta
\end{array}\right]^{\mathrm{T}},
$$

where $r$ and $H$ are obtained through the acoustic image, and are shown in Figure 4 a). $\beta$ is the angle that the sonar beam makes with the line orthogonal to the floor plan, figure 10 .

The line equation in the world reference frame is equal to:

$$
S=m E+b
$$

The point $X i$ in world coordinates its equal:

$$
X_{\text {World }}=x_{v}+R_{v} X_{i} \text {, }
$$

where $R_{v}$ is the rotation matrix from the AUV to world frame. That matrix is given by:

$$
R_{v}=\left[\begin{array}{ccc}
c \psi c \theta & -s \psi & c \psi s \theta \\
s \psi c \theta & c \psi & s \psi s \theta \\
-s \theta & 0 & c \theta
\end{array}\right]
$$

Thus, the coordinates of the world represented in the coordinates of the submarine are:

$$
X i=R_{v}^{-1}\left(X_{\text {world }}-x_{v}\right)
$$

Through (11), (12), (13) and (14) equations, the estimated $s_{T}$ is given by:

$$
s_{T}=\frac{m\left(E v+h_{T} c \psi_{v} s \theta_{v}\right)-h_{T} s \psi_{v} s \theta_{v}+b-S_{v}}{c \psi_{v}+m s \psi_{v}}
$$

The estimated $h_{T}$, is equals to:

$$
h_{T}=\frac{H_{v}}{c \theta_{v}}
$$

So the estimated value of $H$ is given by the following expression:

$$
H=\frac{H_{v}}{c \theta_{v} c \beta}
$$

The observation noise is represented by a zero mean vector and covariance error $\mathrm{R}$ :

$$
\begin{aligned}
& v=\left[\begin{array}{llll}
\epsilon_{s_{T}} & \epsilon_{H} & \epsilon_{\psi_{v}} & \epsilon_{\theta_{v}}
\end{array}\right]^{T} \\
& \mathrm{E}\left[v(k)=[0] \text { and } E\left[v(k) v^{T}(k)\right]=R\right.
\end{aligned}
$$

where $\epsilon_{\psi_{v}}$ and $\epsilon_{\theta_{v}}$ are measurement errors of the AUV yaw and pitch, and $\epsilon_{s_{T}}$ and $\epsilon_{H}$ are derived from the measurement of $s_{T}$ and $H$ in the acoustic image.

Therefore, the observations of the filter are given by the expression:

$$
z(k+1)=\left[\begin{array}{c}
s_{T}+\epsilon_{s_{T}} \\
H+\epsilon_{H}
\end{array}\right]
$$




\section{Kalman Filter: State (Distance, Depth)}

The vehicle state will be defined by the distance of the vehicle to the feature, by the depth coordinated of the vehicle, i.e. the relative height to the sea floor, and the orientation of the feature in the world reference frame. Thus, the state will be:

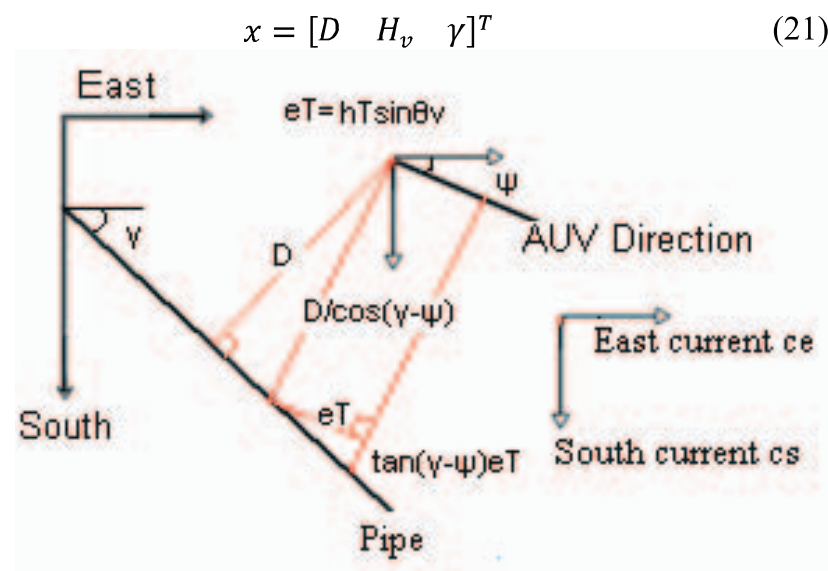

Figure 8 Distance (D) between AUV and pipe.

Taking into account the sea currents in the east and south axis $\left(c_{e}\right)$ and $\left(c_{s}\right)$, the speed of vehicle $\left(V_{e}\right)$ and $\left(V_{h}\right)$, assuming a roll $(\Phi)$ close to zero, and finally taking into account the yaw $(\psi)$ and pitch $(\theta)$ angles, we have:

$$
\begin{aligned}
& \dot{e}_{v}=c \theta(\mathrm{t}) V_{e}(t)+\mathrm{s} \theta(\mathrm{t}) V_{h}(t) \\
& \dot{h}_{v}=\mathrm{s} \theta(\mathrm{t}) V_{e}(t)-\mathrm{c} \theta(\mathrm{t}) V_{h}(t)
\end{aligned}
$$

The vector $u(t)$ corresponds to the system input, and $w(t)$ the white noise. There are the following vectors:

$$
\begin{gathered}
u(t)=\left[\begin{array}{llllll}
V_{e} & V_{h} & \psi & \theta & c_{e} & c_{s}
\end{array}\right]^{T} \\
w(t)=\left[\begin{array}{llllll}
\varepsilon_{V e} & \varepsilon_{V h} & \varepsilon_{\psi} & \varepsilon_{\theta} & \varepsilon_{c e} & \varepsilon_{c s}
\end{array}\right]^{T} \\
E[w(k)]=0 \\
\text { and } E\left[w(k) w^{T}(k)\right]=Q
\end{gathered}
$$

Through figure 8 , the approximation speed from the vehicle to the pipe can be computed and is given by:

$$
\dot{D}=\mathrm{s}(\gamma-\psi) \cdot\left(V_{e} \mathrm{c} \theta+V_{h} \mathrm{~s} \theta\right)-c_{s} \mathrm{c} \gamma+c_{e} \mathrm{~s} \gamma
$$

D. Sonar observations: Feature pipe

In this case, we use three observations: the minimum and maximum distance of the vehicle to the pipe, (Dmin,Dmax) and finally the detected distance, in the acoustic image, between vehicle and the sea bed $(H)$.

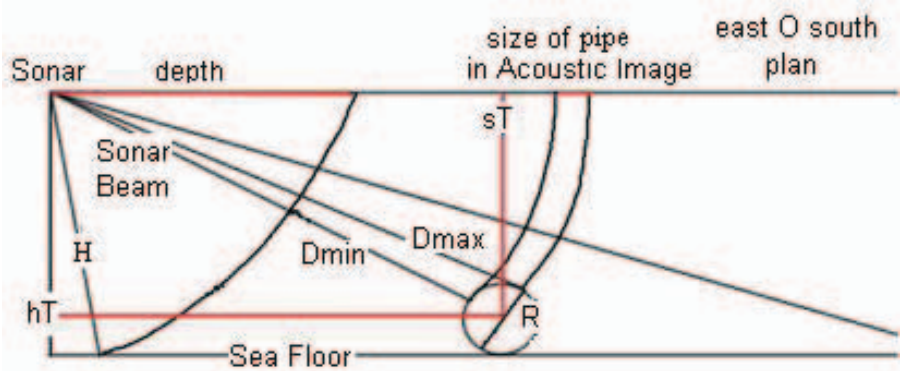

Figure 9 Three observations, Dmin, Dmax and h. Size of pipe in the image.
The minimum distance is related to the system state as follows:

$$
\operatorname{Dmin}=-R+\sqrt{{h_{T}{ }^{2}+s_{T}{ }^{2}}^{2}}
$$

The maximum distance, taking into accounts that the sonar ever sees approximately a circle, is related with the state the following way:

$$
D \max ^{2}=R^{2}+{h_{T}}^{2}+{s_{T}}^{2}
$$

Finally, the third observation $H$ already was computed in previous (17).

The expression for $s_{T}$, also already been computed (15), in order to other states, $E_{v}, S_{v}, H_{v}, m e b$, it will be again compute, now in order to $D, H_{v}$ e $\gamma$ states.

$$
s_{T}=\frac{D}{c(\gamma-\psi)}+\tan (\gamma-\psi) \tan \theta_{v}\left(H_{v}-R\right)
$$

Also $h_{T}$ was been calculated in previous (16), but it differs when radius pipe, $R$, is considered. So $h_{T}$ come:

$$
h_{T}=\frac{H_{v}-R}{\mathrm{c} \theta_{v}}
$$

Thus, the filter observation is given by:

$$
z(k+1)=\left[\begin{array}{c}
D \min +\epsilon_{D \min } \\
D \max ^{2}+\epsilon_{D \max ^{2}} \\
H+\epsilon_{H}
\end{array}\right]
$$

\section{E. Sonar observation: Feature wall}

The wall will be seen by the sonar as a growing strength echo from the minimum distance between the submarine and the wall.

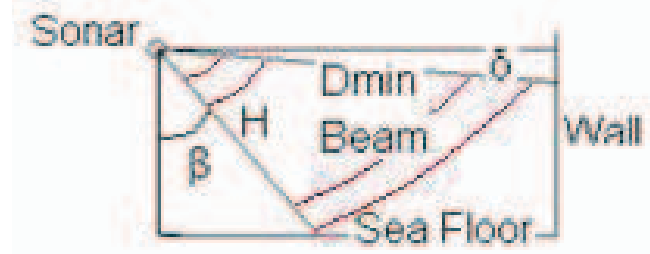

Figure 10 Two observations, Dmin and $\mathrm{H}$, when the feature is a wall.

The filter observation will be formed by the following two characteristics:

$$
z(k+1)=\left[\begin{array}{c}
D \min +\epsilon_{D \min } \\
H+\epsilon_{H}
\end{array}\right]
$$

First observation, Dmin, is determined geometrically by examining figure 11 :

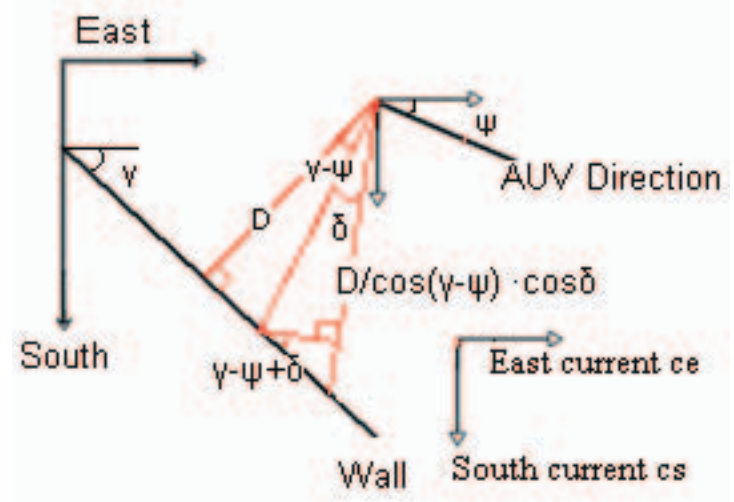

Figure 11 Distance (D) between AUV and wall. 
Resulting in:

$$
\operatorname{Din}=\frac{D}{c(\gamma-\psi)} \cdot(c \delta+s \delta \tan (\gamma-\psi+\delta))
$$

\section{SIDE SCAN OPERATION LIMITS}

Any sonar has a maximum range and it cannot probe the environment at distances higher than such range.

So, when the sonar used is side scan sonar and the need is to follow features like an underwater pipe on the sea floor or a wall, these features cannot for any instant disappear from the range of sonar.

Therefore in this section the side scan operation limits are shown.

The sonar used on this work is the Imagenex SportScan and it can be configured with two frequency modes. When the mode is low frequency $(330 \mathrm{Kz})$ the incidence cone of the acoustic impulses is 60 degrees. But, when the high frequency $(800 \mathrm{Khz})$ mode is chosen for sonar acquisition the incidence angle is 30 degrees.

Still about the sonar beam, the middle line of sonar beam makes 20 degrees with the horizontal line. The next figure shows the sonar beam angles decomposition for low and high frequency modes.

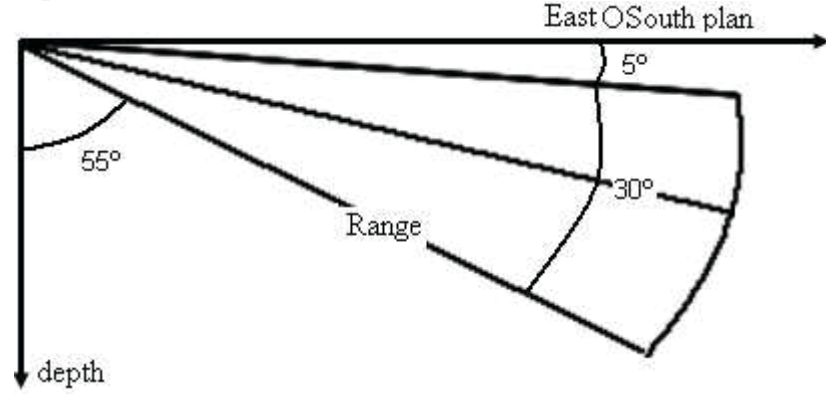

Figure 12 Sonar beam for frequency mode $800 \mathrm{KHz}$ Incident angle of $30^{\circ}$.

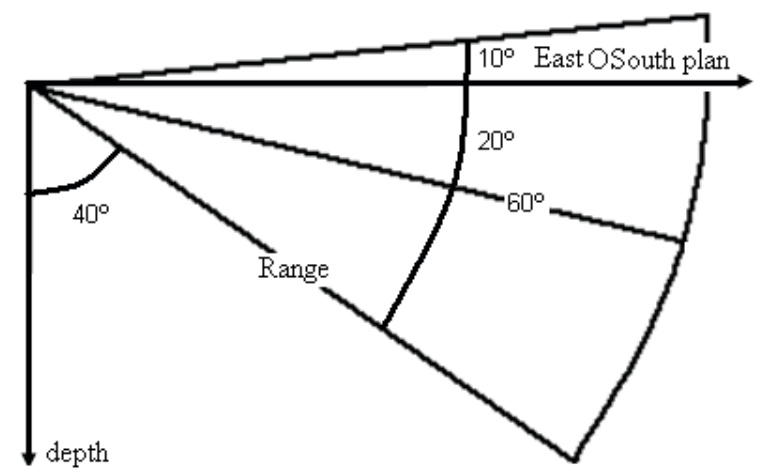

Figure 13 Sonar beam for frequency mode $330 \mathrm{KHz}$. Incidence incident angle of $60^{\circ}$.

For the determination of operation limits of side san sonar we need to consider that on acoustic image the sea floor echo would appear before the feature echo.

The next figure shows the operation zone of sonar, for an underwater pipe feature, when the sonar incidence angle is 30 and 60 degrees and when the range of sonar is 15 meters.
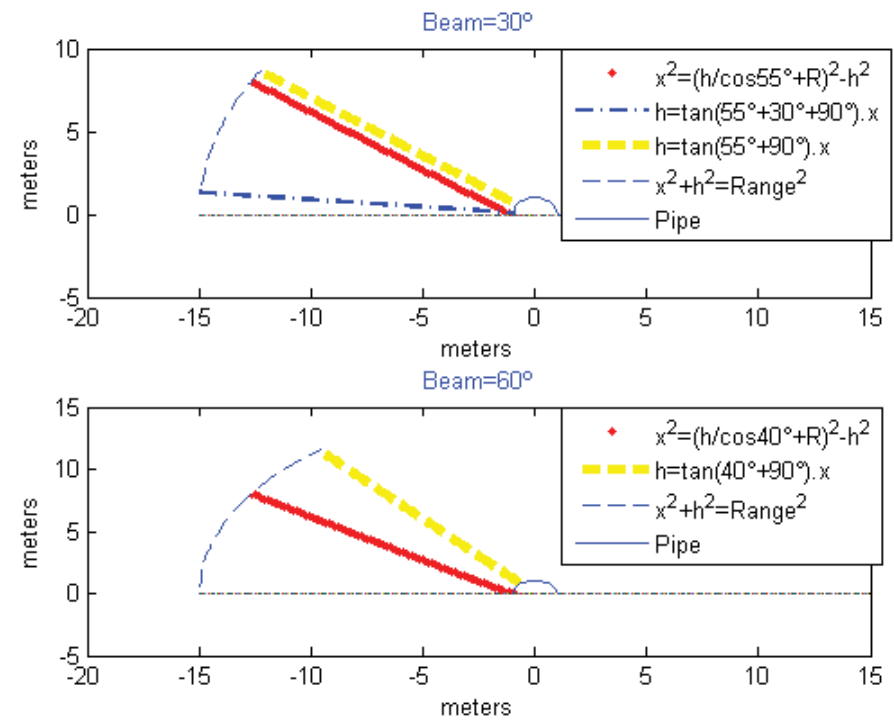

Figure 14 Sonar operations limits for an incident beam of $30^{\circ}$ and when the characteristic seen is a underwater pipe on the sea floor.

\section{AUV GUIDANCE AND CONTROL}

To assess the performance of the developed navigation algorithms, we simulated the whole system with synthesized acoustic images. AUV guidance and control loops were designed, to ensure that the AUV could follow the detected feature.

The fundamental aim is to make the vehicle follow a path parallel to the feature, either the wall or pipe, at a given reference distance Dref. The output of the control is the yaw rate.

The control consist of two PIDs, the first is a function of the distance $f(D-D r e f)$, while the other PID is function of difference between the feature and yaw vehicle angles $f(\gamma-\psi)$. The two PIDs have therefore contrary effects; one tends to increase the relative angle between vehicle and feature. The other tends to decrease this angle. Thus, the second PID is of lower magnitude than the first.

\section{A. Determination of control gains}

The system is not linear and that prevents us from obtaining the control gains in the same way as the linear systems are obtained.

Nonetheless, since the fundamental purpose for this paper is not the application of guidance laws, we followed a somehow empirical procedure for the determination of the control gains.

When the linear speed is higher, the magnitude of the control gains would be also higher so as to keep same trajectory and curvature that is reached when the speed is smaller.

The demonstration of this result is made below. The yaw rate is given by:

$$
\frac{d \psi}{d t}=\frac{d \psi}{d s} \cdot \frac{d s}{d t}=\frac{d \psi}{d s} \cdot v,
$$

where $s$ is the path followed, and $\frac{d s}{d t}$ is the linear speed of the vehicle. Then: 


$$
\frac{d \psi}{d s}=\lim _{\Delta s \rightarrow 0} \frac{\Delta \psi}{\Delta s}=\text { curvature }(k)=\frac{1}{R},
$$

where $\mathrm{R}$ is the radius of curvature of the vehicle trajectory. Thus, for a given curvature, the speed of rotation of yaw will grow proportionally with the linear speed of the vehicle.

The control only enters into effect when distance between the feature and the vehicle is below a certain threshold $(T)$.

For distances greater than $T$, the vehicles approaches the features with a predefined angle $(\alpha)$. Thus, the initial value of the yaw rate control is given by:

$$
\dot{\psi}=(T . m \text { dist }-\alpha . m a n g) v,
$$

where $v$ is the vehicle linear speed.

Considering only the distance proportionally constant, mang equals to zero, for a desired curvature equals to $k_{\text {dist }}$ :

$$
\text { mdist }=\frac{k_{\text {dist }}}{T}
$$

Considering now the equation (37), and constant proportional to distance (38), the angle constant proportional, for a desired curvature of $k$, is:

$$
\operatorname{mang}=\frac{k_{\text {dist }}-k}{\alpha}
$$

So the expression of control path is given by:

$$
\frac{d \psi}{d t}=(\operatorname{mdist}(D-D r e f)-\operatorname{mang}(\gamma-\psi)) v
$$

VIII. Simulation RESUlts

Simulation results are obtained for Kalman filter when the state variables are: the orthogonal distance between the vehicle and the feature, vehicle depth and feature orientation on the world frame.

The simulation time corresponds to a displacement of the vehicle during 3 minutes. In acoustic sensor and the image obtained is introduced random error with mean zero like the reality.

The sensors simulated are the digital compass that measures the yaw, pitch and roll of vehicle on the world benchmark. Also the measurement of sea currents, the east and depth speeds were simulated. On all that measurements overdimensioned errors are introduced.

All vehicle sensors have a $20 \mathrm{~Hz}$ sample rate, while the observation by sonar, is simulated for a $5 \mathrm{~Hz}$ sample rate.

TABLE 1

SIMULATION PARAMETERS.

\begin{tabular}{|c|c|c|}
\hline Feature & Wall & Pipe \\
\hline East/depth Speed $(\mathrm{m} / \mathrm{s})$ & $0.5 / 0$ & $1 / 0$ \\
\hline East/South Corrent $(\mathrm{m} / \mathrm{s})$ & $0 / 0$ & $0.3 / 0.3$ \\
\hline True Orientation (Degrees) & 15 & 30 \\
\hline Reference Distance (meteres) & 7 & 6 \\
\hline
\end{tabular}
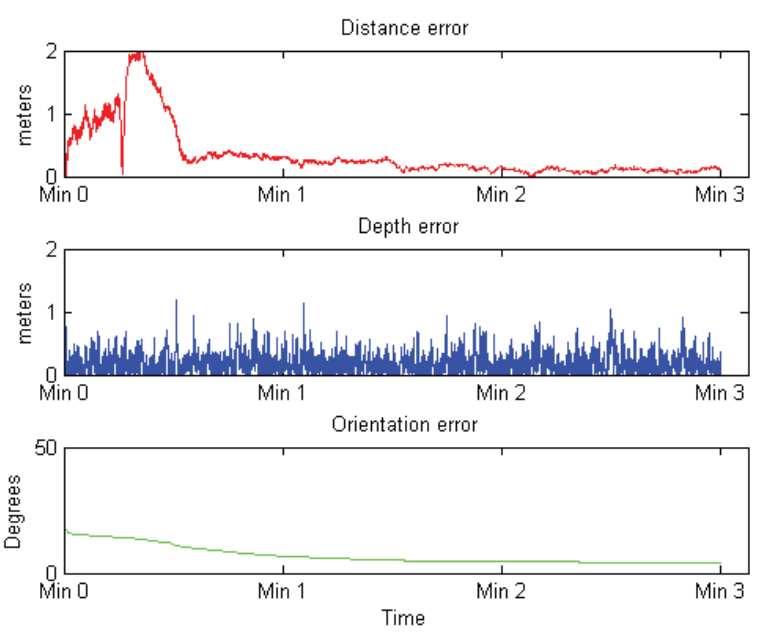

Figure 15 Simulation when the feature is a wall, state error.

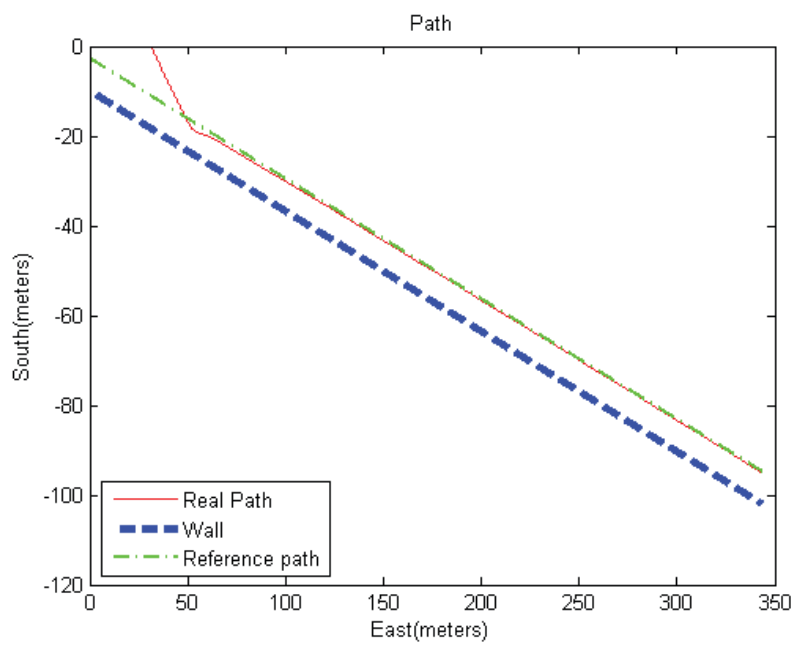

Figure 16 Simulation when the feature is a wall, real and reference path that is a 7 meters parallel path between AUV and the feature.
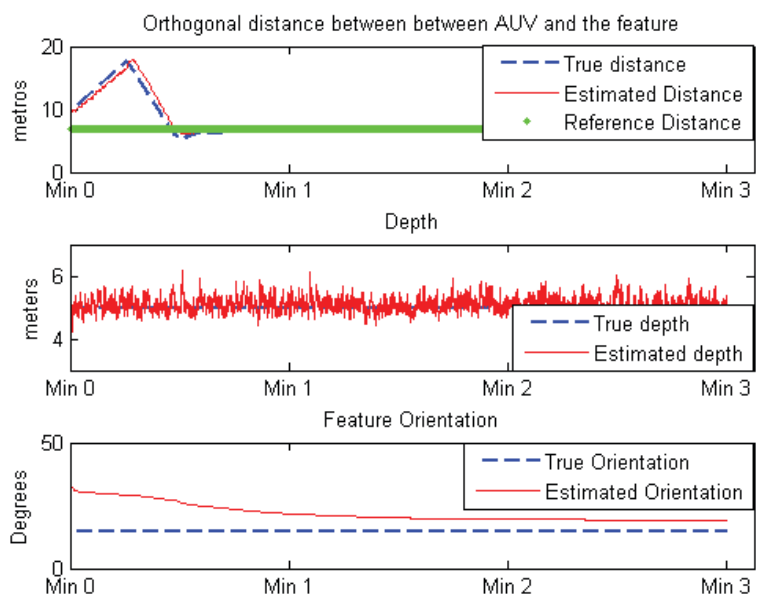

Figure 17 The state variables. 

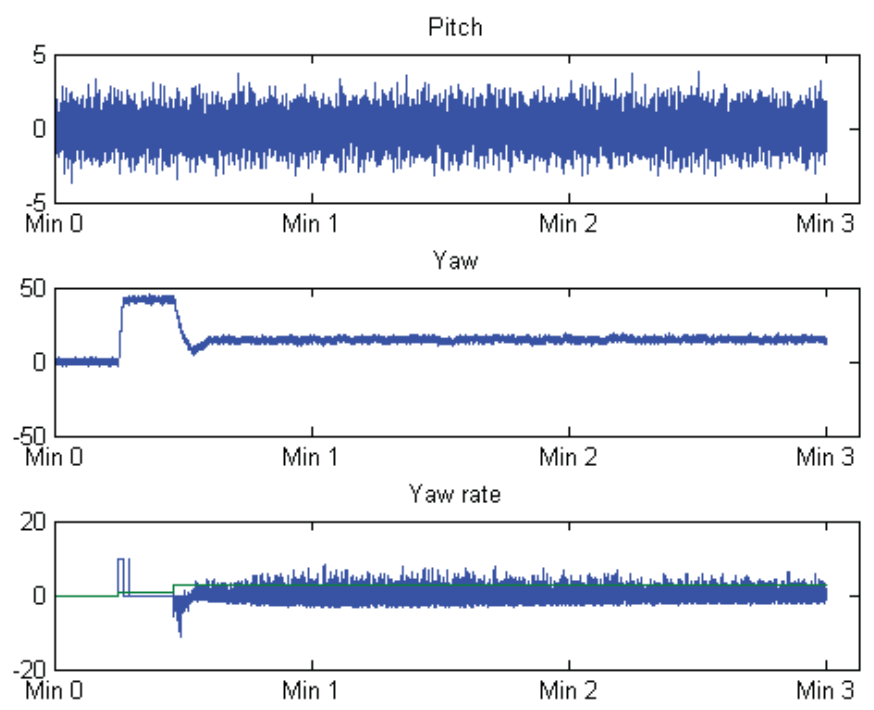

Figure 18 Measured pitch (top) and yaw (middle) on the simulation affected with random noise of zero mean and $1^{\circ}$ of deviation. The bottom graphics show the yaw velocity that the path control gives.

On figure 15 (difference between true state and estimated error) can be seen that error goes to a stationary small error.

The distance error (between a wall and the AUV) in steady state is around $15 \mathrm{~cm}$. The error of maximum depth reached is about 1 meter. The error of angle at steady state goes to 0 .

The figure 16 shows the reference and real path that AUV follows. Can be seen that both are very close, due the good estimation provided by the navigation filter implemented. That also proves that the dimensioned control works.

With red color, on figure 17 , are shown the estimated state variables. With blue color the real state is presented. For the orthogonal distance between the vehicle and the vertical wall the reference distance at green is shown. The real state and estimated are very close.
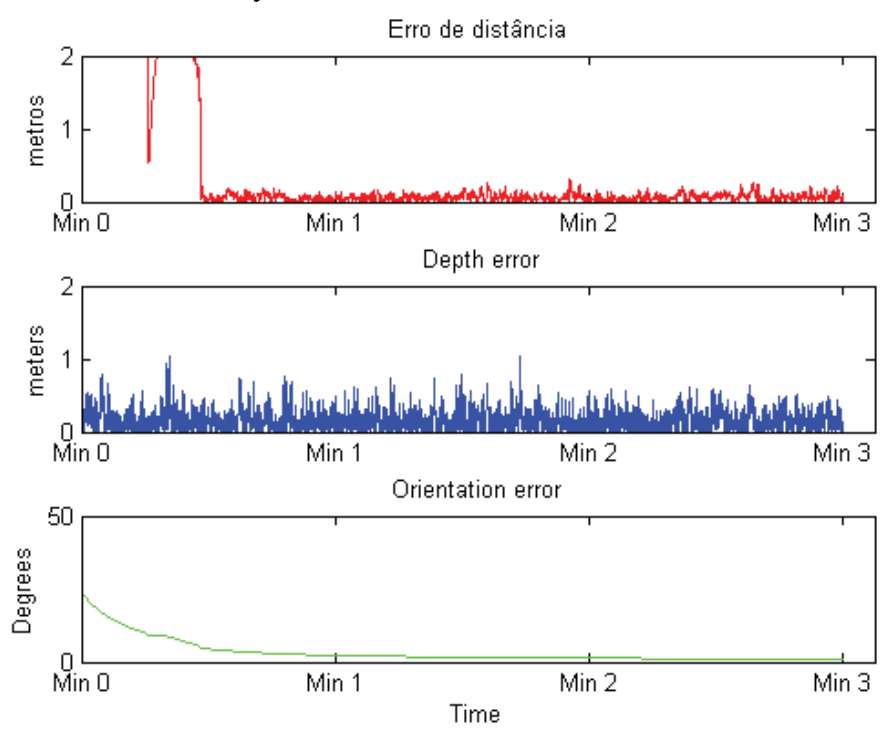

Figure 19 Simulation when the feature is an underwater pipe on the floor. State error.

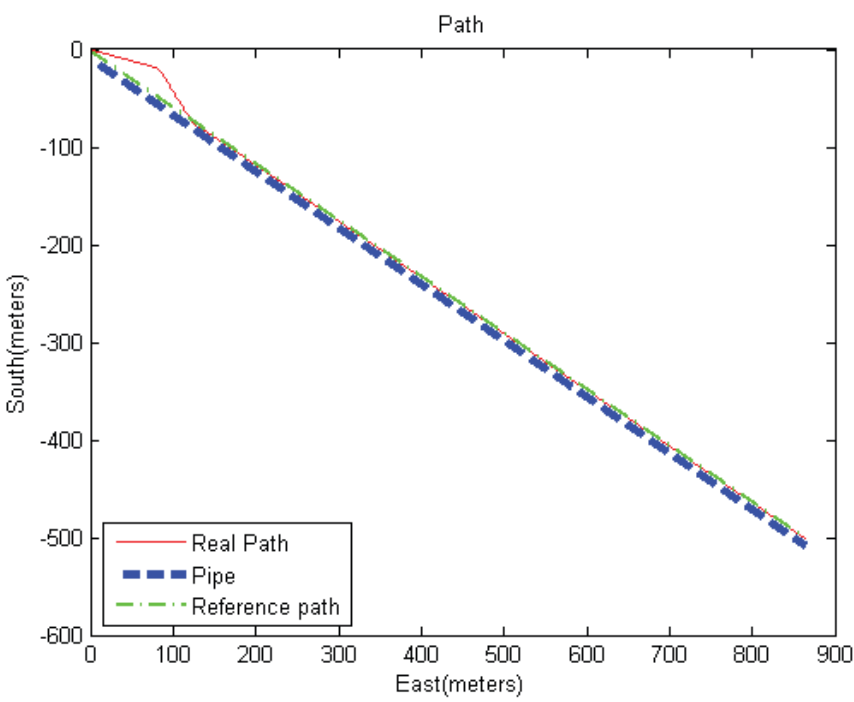

Figure 20 Simulation when the feature is a underwater pipe, real and reference path that is a 6 meters parallel path between AUV and the feature.
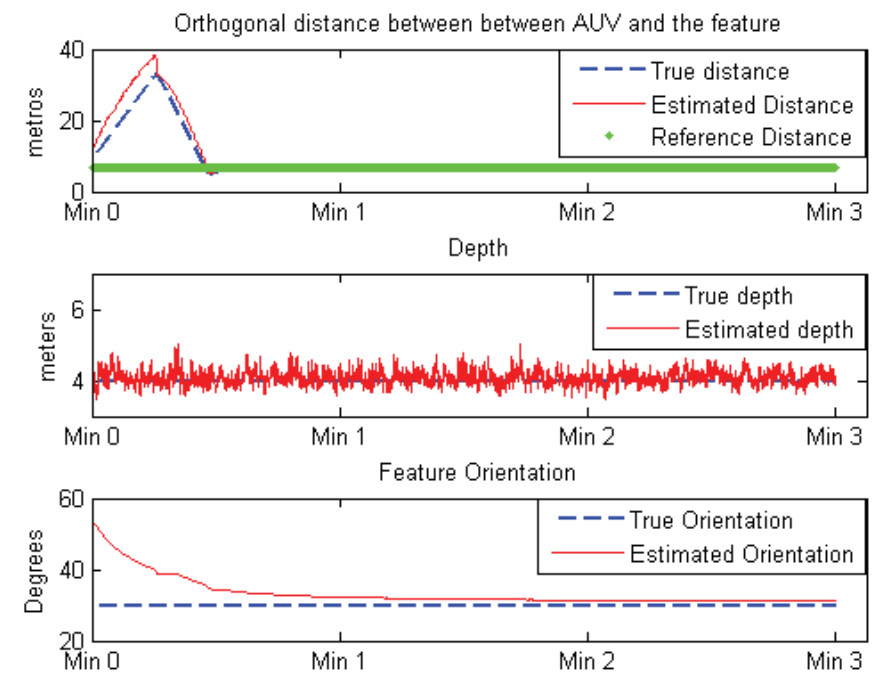

Figure 21 Simulation when the feature is a pipe. Estimated State and the true state, both are close.

\section{CONCLUSIONS}

This paper describes a method for the estimation of the vehicle position with simultaneously map construction. The method is based on Extended Kalman Filter.

Positioning data is provided by side scan sonar and environment observations from two different features of the marine environment are considered: a pipe and a wall. In this work is shown how can be integrated the information from the sensory part of the vehicle.

The control, for the vehicle follow a parallel path to the feature is successful applied on simulation.

This work shows the operation of two Kalman filters implemented. The conclusion acquired was that; the filter in which the vehicle state is the distance to the feature, the 
distance to the sea floor and orientation of the landmark, is the ideal filter and ensures a good estimation.

An interface in $\mathrm{C}++$ has been developed for communication between the AUV and Imagenex SportSan sonar.

An algorithm, that allows extract features from acoustic images and give a numerical representation from the distance to the sea bottom and to features like underwater pipe or a vertical wall, was implemented and validated with a real acoustic image acquired during a mission on the Douro River, Porto.

In future, it is intended to fuse the filter, the control path, the sonar acquisition and the acoustic image segmentation in order to obtain the observations and features values, on a single application in $\mathrm{C}++$ that allows for the AUV follow a parallel path at a reference path from a feature like a pipe or a wall. This future procedure that joins all partial implementations on a single application is described with figure.

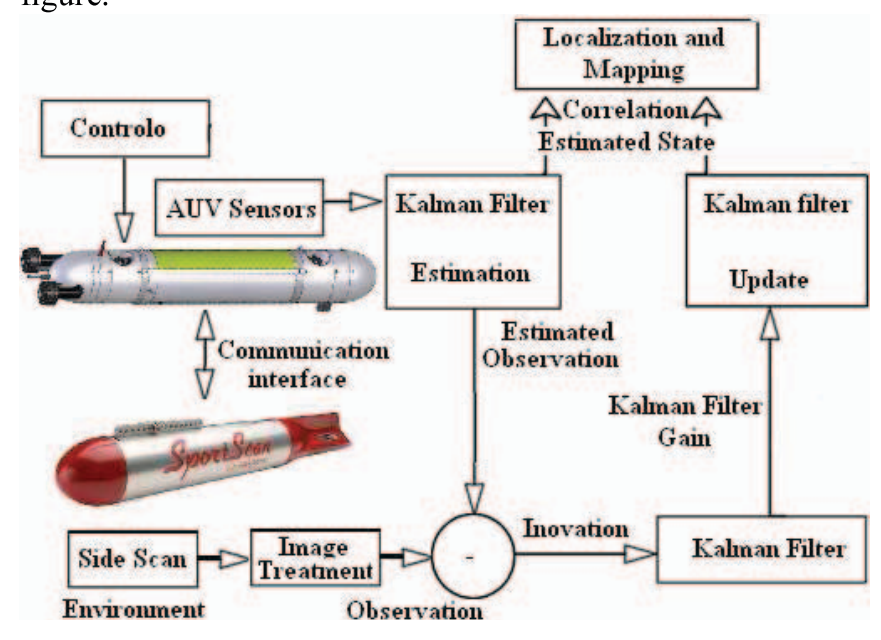

Figure 22 All Project description.

\section{REFERENCES}

[1] M. Breivik and T. I.Fossen, "Guidance Laws for Autonomous Underwater Vehicles" on "Underwater vehicles", ISBN 978-953-761949-7, Publisher: IN-TECH, January 2009.

[2] Wood, Stephen, "Autonomous Underwater Gliders", on "Underwater vehicles", ISBN 978-953-7619-49-7, Publisher: IN-TECH, January 2009.

[3] A. Matos and N. Cruz, "Mares-Navigation, control and On-board Software", on "Underwater vehicles", ISBN 978-953-7619-49-7, Publisher: IN-TECH, January 2009.

[4] B. Bingham, "Navigating Autonoumous Underwater Vehicles", on "Underwater vehicles", ISBN 978-953-7619-49-7, Publisher: IN-TECH, January 2009.

[5] P. E. Hagen,O. Hegrenaes, B. Jalving, O. Midtgaard, M. Wiig and O. K. Hagen, "Making AUVs truly Autonoumous",on "Underwater vehicles", ISBN 978-953-7619-49-7, Publisher: IN-TECH, January 2009.

[6] Michael Scorba, "Simultaneous Localisation and Map Building", Robotic Reacherch Group Department of Engeneering of Oxford, Oxford England, thesis for the Degree of Philosophy, 1997.

[7] J. Borenstain, H. R. Everett, L.Feng and D. Wehe, "Mobile Robot Positioning \& Sensors and Techniques", Invited paper for the Journal of Robotic Systems, Special Issue on Mobile Robots, Vol. 14 No. 4, pp. 231 -249 .

[8] I. T. Ruiz, S. de Raucourt, Y. Petillot, and D. M. Lane, "Concurrent Mapping and Localization Using Sidescan Sonar", IEEE Journal of Oceanic Engineering, Vol. 29 No. 2, April 2004.
[9] A. Gelb, J. F. Kasper Jr., R. A. Nash Jr., C.F. Price and A.A. Sutherland Jr., " Applied Optimal Estimation", The MIT Press, $4{ }^{\text {th }}$ printing, 1996.

[10] I. T. Ruiz, Y. Petillot and D. M. Lane, "Improved AUV Navigation using Side-scan Sonar", Ocean Systems Laboratory School of Engineering and Physical Sciences Heriot-Watt University, Scotland, UK.

[11] P.Newman H.Durrant-Whyte, "Using Sonar in Terrain-Aided Underwater Navigation", International Conference on Robotics \& Automation Leuven, Belgium, May 1998.

[12] D.E. Di Massa and W.K. Stewart Jr "Terrain-Relative Navigation for Autonomous Underwater Vehicles", Woods Hole Oceanographic Institution.

[13] Imagenex Sportscan sonar datasheet. It can be found in the web site: $\mathrm{http}: / / \mathrm{www}$. imagenex.com/Downloads/downloads.html. Accessed on:20 August 2009

[14] R. C. Gonzalez and R. E. Woods "Digital Image Processing", ISBN 0201-50803-6, September 1993.

[15] K. R. Castleman, "Digital Image Processing", ISBN 0-13-211467-4, December 1996.

[16] S. E. Umbaugh, "Computer Vision and Image Processing", ISBN 0-13264599-8, June 1999.

[17] T. I. Fossen, "Guidance and Control of Ocean Vehicles", ISBN 0-47194113-1, December 1995. 